\title{
Correlación de diferentes métodos de valoración de la rigidez matinal con índices de actividad y discapacidad en pacientes con artritis reumatoidea
}

\author{
B.I. Barrios ${ }^{1}$, S.B. Papasidero ${ }^{1}$, M.A. Medina ${ }^{1}$, R.E. Chaparro del Moral ${ }^{1}$, O.L. Rillo ${ }^{2}$, S.O. Paira ${ }^{3}$, C. Sandobal ${ }^{3}$ \\ ${ }^{1}$ Servicio de Reumatología, Hospital General de Agudos Dr. E. Tornú, CABA; ${ }^{2}$ Sección de Reumatología del Hospital I. Pirovano, CABA; y \\ ${ }^{3}$ Sección de Reumatología, Hospital. J.M. Cullen, Santa Fe.
}

\section{Resumen}

La rigidez matinal (RM) es un síntoma complejo en los pacientes con AR. Existen diferentes formas de valorar la RM. Sin embargo, la mejor manera de evaluarla es aún controversial.

Objetivos: Comparar la severidad y la duración de la RM, evaluada por el médico y por cuestionarios autorreportados, con la actividad de la enfermedad y la capacidad funcional en pacientes con AR.

Métodos: Estudio observacional de corte transversal. Se incluyeron pacientes con diagnóstico de AR. Se consignaron datos demográficos, características de la AR y medidas clinimétricas. Los pacientes fueron asignados aleatoriamente a uno de dos grupos: el primer grupo fue interrogado sobre la duración de la RM en minutos; el segundo grupo fue evaluado mediante un cuestionario autorreportado que cuantificaba la severidad de la RM (EVA numérica y no numérica) y la duración (en minutos y en escala de Likert).

Resultados: Participaron 111 pacientes (86\% mujeres). La edad media fue de 52 años y la mediana de duración de la enfermedad fue 8 años. El 51\% presentaba RM. La correlación de la RM en minutos medida por el médico fue buena con HAQ-A y aceptable con DAS28 y CDAl; mientras que la severidad medida por EVA correlacionó mejor con los índices de actividad de la AR que con discapacidad. Cuando la intensidad de la RM fue evaluada por EVA se observó que un valor $>5,5 \mathrm{~cm}$ se asoció con actividad severa de la enfermedad (Sensibilidad: 44\%; Especificidad: 100\%).

Conclusión: La duración de la rigidez matinal, evaluada en minutos por el médico, estaría relacionada tanto con la actividad de la enfermedad como con la discapacidad. La intensidad de la rigidez matinal se asoció más fuertemente con la actividad inflamatoria en los pacientes con AR.

\section{Abstract}

Morning stiffness (MS) is a complex symptom in rheumatoid arthritis (RA) patients. There are different ways to assess MS. However, the best way to evaluate it is still controversial.

Objectives: To compare the severity and duration of MS (assessed by the physician and by self-report questionnaires) with disease activity and functional disability in a cohort of RA patients.

Methods: We conducted a cross-sectional observational study. Patients with RA were included. Demographic data, RA characteristics and clinimetric measures were collected. Patients were randomly assigned to one of two groups: the first group was asked about the MS duration in minutes; those in the second group were evaluated using a self-reported questionnaire which included severity (numerical and non-numerical VAS) and duration (in minutes and Likert Scale) of the MS.

Results: One hundred and eleven patients were included $(86 \%$ women). Mean age was 52 years and median disease duration was 8 years. MS was reported by $51 \%$ of the patients. MS measured in minutes by the physician showed a good correlation with HAQ-A and an acceptable correlation with DAS28 and CDAl; MS severity assessed by VAS correlated better with RA activity indexes than with disability. When intensity of MS was evaluated by VAS, we observed that a value $>5.5$ centimeters was associated with severe disease activity (sensitivity $44 \%$ and specificity $100 \%$ ).

Conclusion: MS duration in minutes assessed by the physician would be associated with both disease activity and disability. Intensity of morning stiffness was most strongly associated with inflammatory activity in RA patients.

Key words: rheumatoid arthritis, morning stiffness.

Palabras clave: artritis reumatoidea, rigidez matinal. 


\section{Introducción}

La Artritis Reumatoidea (AR) es una enfermedad inflamatoria sistémica de origen autoinmune, caracterizada por poliartritis simétrica de grandes y pequeñas articulaciones, de evolución crónica y progresiva que puede provocar discapacidad funcional y alteración de la calidad de vida en los pacientes que la padecen $^{1}$.

Uno de los síntomas más característicos es la rigidez matinal (RM). La misma se define como el enlentecimiento o dificultad del movimiento articular al levantarse por la mañana o luego de permanecer en una misma posición por un tiempo prolongado que característicamente mejora con el movimiento. Puede comprometer varias articulaciones a ambos lados del cuerpo produciendo limitación funcional ${ }^{2}$. Suele ser el primer síntoma de presentación de la AR y es considerado un factor de riesgo para el desarrollo de enfermedad más severa. En pacientes con artritis temprana, se ha demostrado que la duración prolongada de la RM (mayor a 60 minutos) es un predictor de enfermedad erosiva $^{3}$. Asimismo, la duración y la severidad de la RM se correlacionan con medidas de actividad de la enfermedad, incluyendo aquellas reportadas por los pacientes ${ }^{4}$.

En cuanto a la fisiopatología de la RM, ésta ha sido asociada al compromiso del ritmo circadiano secundario a alteraciones a nivel del eje hipotálamo-hipófiso-adrenal. Se ha postulado que niveles elevados de citoquinas proinflamatorias, como IL-6 y TNF-alfa, producirían un pico de cortisol sérico más temprano al fisiológico y, por lo tanto, niveles inapropiadamente bajos para el grado de inflamación que se produce durante la mañana ${ }^{5}$. Buttgereit y cols., en el año 2008, observaron una reducción significativa de la RM en aquellos pacientes a los cuales se les administró prednisona de liberación prolongada antes de acostarse (alcanzando su pico máximo 4 horas luego de su ingesta), en comparación con aquellos que recibieron prednisona a la mañana en forma convencional, sin modificar otras variables en relación a la actividad de la enfermedad ${ }^{6}$.

Existen diferentes formas de valorar la RM. En la mayoría de los estudios clínicos, la RM se expresa en minutos, interrogando al paciente sobre su duración desde que se levanta hasta que alcanza su máxima mejoría. Otra forma de evaluar la RM es teniendo en cuenta la severidad de la misma, lo cual puede realizarse por medio de una escala visual analógica (EVA) ${ }^{7}$. La severidad de la RM ha demostrado presentar menor variabilidad que su duración. Además, la evaluación de la RM medida mediante una EVA de severidad demostró diferenciar mejor entre pacientes con enfermedad activa en comparación con aquellos en remisión. Por lo tanto, la evaluación de la severidad de la RM sería más sensible para detectar cambios clínicos significativos con respecto a la medición de su duración ${ }^{8}$. Sin embargo, la mejor manera de evaluar la RM continúa siendo motivo de controversia?.

Por todo lo expuesto, el objetivo de nuestro trabajo fue comparar la severidad y la duración de la RM (evaluada por el médico y mediante un cuestionario autorreportado), con la actividad de la enfermedad y la capacidad funcional en una cohorte de pacientes con AR.

\section{Material y métodos}

Se realizó un estudio observacional, analítico, de corte transversal. Se incluyeron pacientes consecutivos $\geq 18$ años de edad, con diagnóstico establecido de AR según criterios ACR $1987^{10}$ de dos centros de reumatología de Argentina, que aceptaron participar mediante la firma de un consentimiento informado. Se excluyeron pacientes con alguna condición que impidiera la lectura o comprensión de los cuestionarios.

Los pacientes fueron divididos en forma aleatoria en dos grupos: Grupo 1: se les interrogó sobre la duración de la RM en minutos (desde que se levantaba por la mañana hasta que alcanzaba la máxima mejoría); Grupo 2: se evaluó la RM mediante un cuestionario autorreportado que valoró tanto la severidad de la misma (mediante EVA numérica y no numérica) como su duración (en minutos y mediante escala de Likert). A todos los pacientes se les realizó recuento de articulaciones dolorosas e inflamadas (recuento de 28); y completaron EVA para: dolor (rango 0-10 cm), evaluación global de la enfermedad por el paciente y por el médico (rango 0-10 $\mathrm{cm}$ ) y HAQ- ${ }^{11}$. Se consignó el valor de eritrosedimentación (ESD) mediante método de Westergreen. Con los datos obtenidos se calcularon los índices compuestos DAS28 y $\mathrm{CDAI}^{12}$. Todas las evaluaciones se llevaron a cabo en la misma visita y durante la mañana.

Análisis estadístico: Se describieron las características poblacionales sociodemográficas y de la enfermedad con medias, medianas, desvío estándar (DE) y rango intercuartílico (RIC) según correspondía. La correlación entre los parámetros clínicos y la RM se determinó mediante coeficiente de correlación de Pearson (r). Se consideró un valor de $r>0,5$ como buena correlación. También se llevó a cabo el modelo de regresión lineal, para el cual los pacientes sin RM fueron excluidos de este análisis. Mediante curvas ROC se calculó la sensibilidad y especificidad y el área 
bajo la curva (AUC) a fin de hallar un valor de RM que mejor se relacione con la gravedad de la AR.

\section{Resultados}

Se incluyeron en total 111 pacientes con AR, de los cuales el $86 \%$ eran mujeres. La media de edad fue de 52 años (DE 13) y la mediana de tiempo de evolución de la enfermedad fue de 8 años (RIC 4-12). Las demás características clínicas de la población se resumen en la Tabla 1.

\begin{tabular}{|l|c|}
\hline EVALUACIÓN & MEDIANA (RIC) \\
\hline Recuento de articulaciones dolorosas & $3(0-7)$ \\
\hline Recuento de articulaciones tumefactas & $5,8(1-10)$ \\
\hline $\begin{array}{l}\text { EVA de evaluación global de la enfermedad } \\
\text { por el paciente (en cm) }\end{array}$ & $3,5(1,4-5,3)$ \\
\hline $\begin{array}{l}\text { EVA de evaluación global de la enfermedad } \\
\text { por el médico (en cm) }\end{array}$ & $2,3(1-5)$ \\
\hline RM duración en minutos & $60(20-120)$ \\
\hline ESD en mm/1er hora & $30(16-53)$ \\
\hline CDAl & $14(7,1-30)$ \\
\hline DAS28 & $4,2(3,1-5,5)$ \\
\hline HAQ-A & $0,62(0,2-1,3)$ \\
\hline
\end{tabular}

Tabla 1. Clinimetría de los pacientes evaluados ( $N=111)$.

A 52 pacientes se les interrogó sobre la duración de la RM en minutos (Grupo 1); y 59 pacientes (Grupo 2) fueron evaluados mediante el cuestionario. La RM estuvo presente en 57 pacientes $(57 / 111=51 \%)$ : 27 pertenecían al Grupo 1 y 30 al Grupo 2.

La RM interrogada por el médico tuvo una mediana de 60 minutos (RIC 20-120) y presentó una buena correlación con el HAQ-A $(r=0,72 ; \mathrm{p}<0,001)$, mientras que con el DAS28 y el CDAI mostró una correlación moderada. Se observó una correlación aceptable entre la severidad de la RM evaluada por EVA (escala numérica y no numérica) y el DAS28. (Tabla 2 y Gráfico 1)

\begin{tabular}{|l|l|c|c|c|}
\hline \multicolumn{2}{|l|}{ RIGIDEZ MATINAL } & DAS28 & CDAI & HAQ-A \\
\hline INTERROGATORIO & En minutos & $0,63^{*}$ & $0,60^{\star}$ & $0,72^{*}$ \\
\hline $\begin{array}{l}\text { CUESTIONARIO } \\
\text { AUTORREPORTAD0 }\end{array}$ & En minutos & $0,38^{*}$ & $0,32^{\star}$ & 0,01 \\
\cline { 2 - 5 } & En escala Likert & $0,37^{*}$ & 0,30 & 0,009 \\
\cline { 2 - 5 } & $\begin{array}{l}\text { Intensidad } \\
\text { en EVA no numérico }\end{array}$ & $0,61^{*}$ & 0,55 & $0,33^{*}$ \\
\cline { 2 - 5 } & $\begin{array}{l}\text { Intensidad } \\
\text { en EVA numérico }\end{array}$ & $0,61^{*}$ & 0,57 & 0,30 \\
\hline
\end{tabular}

${ }^{*} \mathrm{p}<0,05$

Tabla 2. Correlación de la RM con DAS28, CDAl y HAQ-A.

En las evaluaciones realizadas por cuestionarios, la RM en minutos y por escala de Likert mostraron valores de correlación más bajos. (Tabla 2)

Al analizar las evaluaciones de la RM mediante las

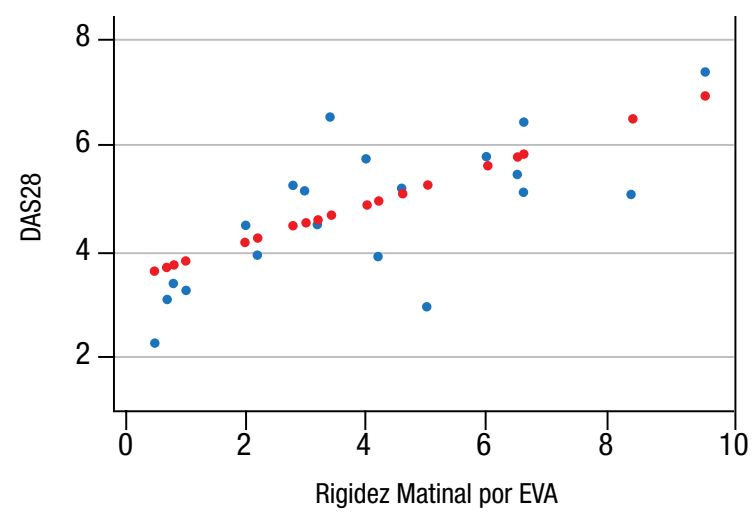

Gráfico 1. Regresión lineal entre DAS28 e intensidad de RM por EVA.

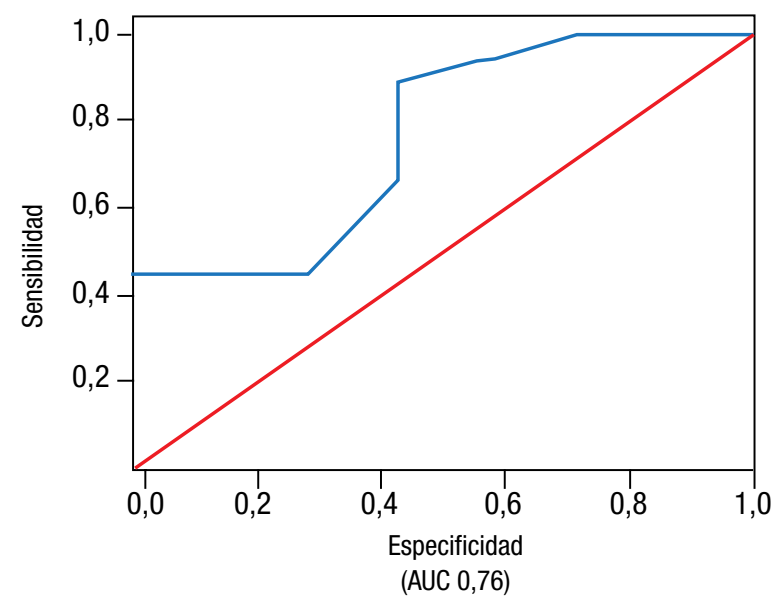

Gráfico 2. Curvas ROC. Actividad de la enfermedad por EVA.

EVA (numéricas y no numéricas), se observó que un valor $>5,5 \mathrm{~cm}$ se asoció con actividad de la enfermedad más grave (DAS28 $>5,1$ ), con una sensibilidad del $44 \%$ y una especificidad del 100\% (AUC 0,76). (Gráfico 2)

\section{Discusión}

En los pacientes con AR, la RM puede dar como resultado una importante limitación en la capacidad funcional, provocando principalmente dificultad para la realización de las actividades de la vida diaria (por ejemplo, levantarse de la cama, asearse o vestirse $)^{2,13}$.

Existen investigaciones que confirman que la disminución en la duración de la RM asociado o no a otros parámetros de actividad de la enfermedad implica una mejoría significativa en la calidad de vida relacionada con la salud ${ }^{6,14}$.

El monitoreo de los pacientes que padecen AR es complejo debido a que no existe un índice compuesto que 
sea adecuado para todos los casos. Algunos de ellos pueden presentar un alto número de articulaciones inflamadas, en otros predomina el dolor, mientras que otros pacientes pueden experimentar a la RM como el síntoma más preocupante de su enfermedad ${ }^{15,16}$. El tratamiento debe incluir entre sus objetivos ayudar al paciente a sentirse mejor, algo que no puede ser detectado solamente a través del examen físico o de estudios complementarios, los cuales subestiman la RM. Por este motivo es que últimamente se ha considerado importante priorizar a este síntoma como un ítem potencial dentro de las medidas reportadas por los pacientes (PROMs: patient-reported outcome measures) $)^{17}$.

A pesar de ser un síntoma cardinal de la AR, es de resaltar que la RM no está incluida en los nuevos criterios de clasificación ACR/EULAR 201018, y tampoco forma parte de la mayoría de los índices de actividad de la enfermedad disponibles en la actualidad. Consideramos importante remarcar que la valoración de la RM está comprendida en el core set de las medidas de brote en AR, alcanzando el $79 \%$ de consenso entre los integrantes del grupo OMERACT ${ }^{19,20}$.

El grupo de pacientes evaluados en nuestra cohorte presenta algunas diferencias considerables con el estudio de Da Mota y cols ${ }^{21}$. Tanto la frecuencia como la duración de la RM fueron mayores en la cohorte brasileña en comparación con nuestro trabajo ( $95 \%$ vs $51 \%$ y mediana de 157 minutos vs 60 minutos, respectivamente). Estas diferencias podrían explicarse a que dicho estudio incluyó sólo pacientes con AR temprana (en quienes la RM es muy importante) y al menor número de pacientes evaluados ( $\mathrm{n}$ $65)$.

En nuestro estudio, la duración de la RM evaluada por el médico a través del interrogatorio, mostró una buena correlación con la discapacidad funcional en los pacientes con AR y moderada con la actividad de la enfermedad. En otros estudios, la RM también ha mostrado una mejor correlación con la discapacidad funcional, el dolor y la evaluación global de la enfermedad por el paciente que con otros marcadores clínicos y de laboratorio tradicionales de inflamación (como el recuento articular y la ESD) ${ }^{4,22}$.

Por otro lado, hallamos que la severidad de la RM evaluada mediante EVA (escalas numéricas y no numéricas) sería un mejor indicador de actividad de la enfermedad, ya que un valor $>5,5 \mathrm{~cm}$ se asoció con un DAS28 $>5,1$, con una especificidad del 100\% (AUC 0,76). Estos datos coinciden con los publicados en trabajos previos en donde la RM evaluada mediante EVA mostró una mejor correlación con la actividad de la AR y la respuesta al tratamiento en comparación con la RM informada en minutos ${ }^{3,4}$.

Destacamos como aspectos positivos de este trabajo el número de pacientes incluidos, asistidos durante la práctica clínica diaria de diferentes ciudades del país (CABA y Santa Fe). Además, es de destacar que las evaluaciones se realizaron en el mismo rango de horario (durante la mañana) y en similares condiciones clínicas en todos los pacientes.

Una de las limitaciones de este trabajo fue que no se evaluó la sensibilidad al cambio de las diferentes formas de valorar la RM (ha sido evidenciado en otros estudios que la valoración de la severidad de la RM mediante EVA sería más sensible al cambio con respecto a su medición en minutos). Otras limitaciones fueron el bajo número de pacientes que informó la presencia de RM (que podría estar relacionado con el tiempo de evolución de la enfermedad) y que no se tuvo en cuenta el tratamiento que recibían los pacientes.

Remarcamos la importancia de considerar la presencia de la rigidez matinal en la evaluación global de los pacientes con AR.

Nuestro trabajo nos permitió evidenciar que, de los diferentes métodos para evaluar la RM en los pacientes con AR, la EVA no numérica sería un mejor indicador de la actividad inflamatoria de la enfermedad, mientras que la RM interrogada en minutos por el médico demostró correlacionar no solo con el grado de actividad sino también con el compromiso funcional que afecta a los pacientes con AR. Por lo tanto, consideramos que esta última podría ser la más adecuada para su uso en la práctica diaria.

\section{Bibliografía}

1. Lee DM, Weinblatt ME. Rheumatoid arthritis. Lancet 2001;358(9285):903-11.

2. Lineker S, Badley E, Charles C et al. Defining morning stiffness in rheumatoid arthritis. J Rheumatol 1999;26(5):1052-7.

3. Visser H, Breedveld FC, Hazes JM et al. How to diagnose rheumatoid arthritis early: a prediction model for persistent (erosive) arthritis. Arthritis Rheum 2002;46(2):357-65.

4. Khan NA, Yazici Y, Calvo-Alen J et al. Reevaluation of the role of duration of morning stiffness in the assessment of rheumatoid arthritis activity. J Rheumatol 2009;36:2435-42.

5. Straub RH, Cutolo M. Circadian rhythms in rheumatoid arthritis: implications for pathophysiology and therapeutic management. Arthritis Rheum 2007;56(2):399-408.

6. Buttgereit F, Doering G, Alten R et al. Efficacy of modified-release versus standard prednisone to reduce 
duration of morning stiffness of the joints in rheumatoid arthritis (CAPRA-1): a double-blind, randomised controlled trial. Lancet 2008;371(9608):205-14.

7. Hazes JM, Hayton R, Burt J et al. Consistency of morning stiffness: an analysis of diary data. Br J Rheumatol 1994;33(6):562-5.

8. Vliet Vlieland TPM, Zwinderman AH, Breedveld FC et al. Measurement of morning stiffness in rheumatoid arthritis clinical trials. J Clin Epidemiol 1997;50(7):757-63.

9. Hazes JM, Hayton R, Silman AJ. A reevaluation of the symptom of morning stiffness. J Rheumatol 1993;20(7):1138-42.

10. Arnett FC, Edworthy SM, Bloch DA et al. The American Rheumatism Association 1987 revised criteria for the classification of rheumatoid arthritis. Arthritis Rheum 1988;31(3):315-24.

11. Citera G, Arriola MS, Maldonado-Cocco JA et al. Validation and crosscultural adaptation of an Argentine spanish version of the health assessment questionnaire disability index. J Clin Rheumatol 2004;10(3):110-5.

12. Aletaha D, Nell VPK, Stamm T et al. Acute phase reactants add little to composite disease activity indices for rheumatoid arthritis: validation of a clinical activity score. Arthritis Res Ther 2005;7(4):R796-R806.

13. Da Silva JAP, Phillips S, Buttgereit F. Impact of impaired morning function on the lives and well-being of patients with rheumatoid arthritis. Scand J Rheumatol 2011;125:6-11.

14. Iqbal I, Dasgupta B, Taylor P et al. Elicitation of health state utilities associated with different durations of morning stiffness in rheumatoid arthritis. J Med Econ 2012;15(6):1192-200.

15. Sokka T. Morning stiffness and other patient-reported outcomes of rheumatoid arthritis in clinical practice. Scand J Rheumatol 2011;(Suppl)125:23-7.

16. Halls S, Dures E, Kirwan J et al. Stiffness is more than just duration and severity: a qualitative exploration in people with rheumatoid arthritis. Rheumatology (Oxford) 2015;54(4):615-22.

17. Kalyoncu U, Dougados M, Daurès JP, et al. Reporting of patient-reported outcomes in recent trials in rheumatoid arthritis: A systematic literature review. Ann Rheum Dis. 2009;68:183-90.

18. Funovits J, Aletaha D, Bykerk V et al. The 2010 American College of Rheumatology/European League Against Rheumatism classification criteria for rheumatoid arthritis: methodological report phase I. Ann Rheum Dis 2010;69(9):1589-95.
19. Bartlett SJ, Hewlett S, Bingham $\mathrm{CO}$ et al. Identifying core domains to assess flare in rheumatoid arthritis: an OMERACT international patient and provider combined Delphi consensus. Ann Rheum Dis 2012;71(11):1855-60.

20. Orbai A, Halls S, Hewlett S et al. More than Just Minutes of Stiffness in the Morning: Report from the OMERACT Rheumatoid Arthritis Flare Group Stiffness Breakout Sessions. J Rheumatol 2015;42(11):21824.

21. Da Mota LM, Laurindo IM, Dos Santos Neto LL. Demographic and clinical characteristics of a cohort of patients with early rheumatoid arthritis. Rev Bras Reumatol 2010;50(3):235-48.

22. Yazici $\mathrm{Y}$, Pincus T, Kautiainen $\mathrm{H}$ et al. Morning stiffness in patients with early rheumatoid arthritis is associated more strongly with functional disability than with joint swelling and Erythrocyte Sedimentation Rate.J Rheumatol 2004;31(9):1723-6. 